\title{
Ion-pairing with triethylammonium acetate improves solid-phase extraction of ADP-ribosylated peptides
}

\author{
Robert Lyle McPherson¹, Shao-En Ong²*, and Anthony K. L. Leung1,3,4* \\ ${ }^{1}$ Department of Biochemistry and Molecular Biology, Bloomberg School of Public \\ Health, Johns Hopkins University, Baltimore, MD 21205, USA \\ 2Department of Pharmacology, University of Washington, Seattle, WA 98195, USA \\ 3Department of Molecular Biology and Genetics, School of Medicine, Johns Hopkins \\ University, Baltimore, MD 21205, USA \\ 4Department of Oncology, School of Medicine, Johns Hopkins University, Baltimore, \\ MD 21205, USA
}

\section{Supporting Information}

\section{Table of Contents}

S1. Title Page

S2. Supplementary Figure 1. Solid-phase extraction with cationic ion-pairing reagents improves recovery of ADP-ribosylated peptides.

(separate file) Supplementary Table 1. List of phosphoribosylated peptides identified by LC-MS/MS with relevant details.

(separate file) Supplementary Table 2. Statistical details of two-sample t-tests on physicochemical properties of phosphoribosylated peptides identified in TFA or TEAA extracted samples. 


\section{Supplementary Figure 1}

A

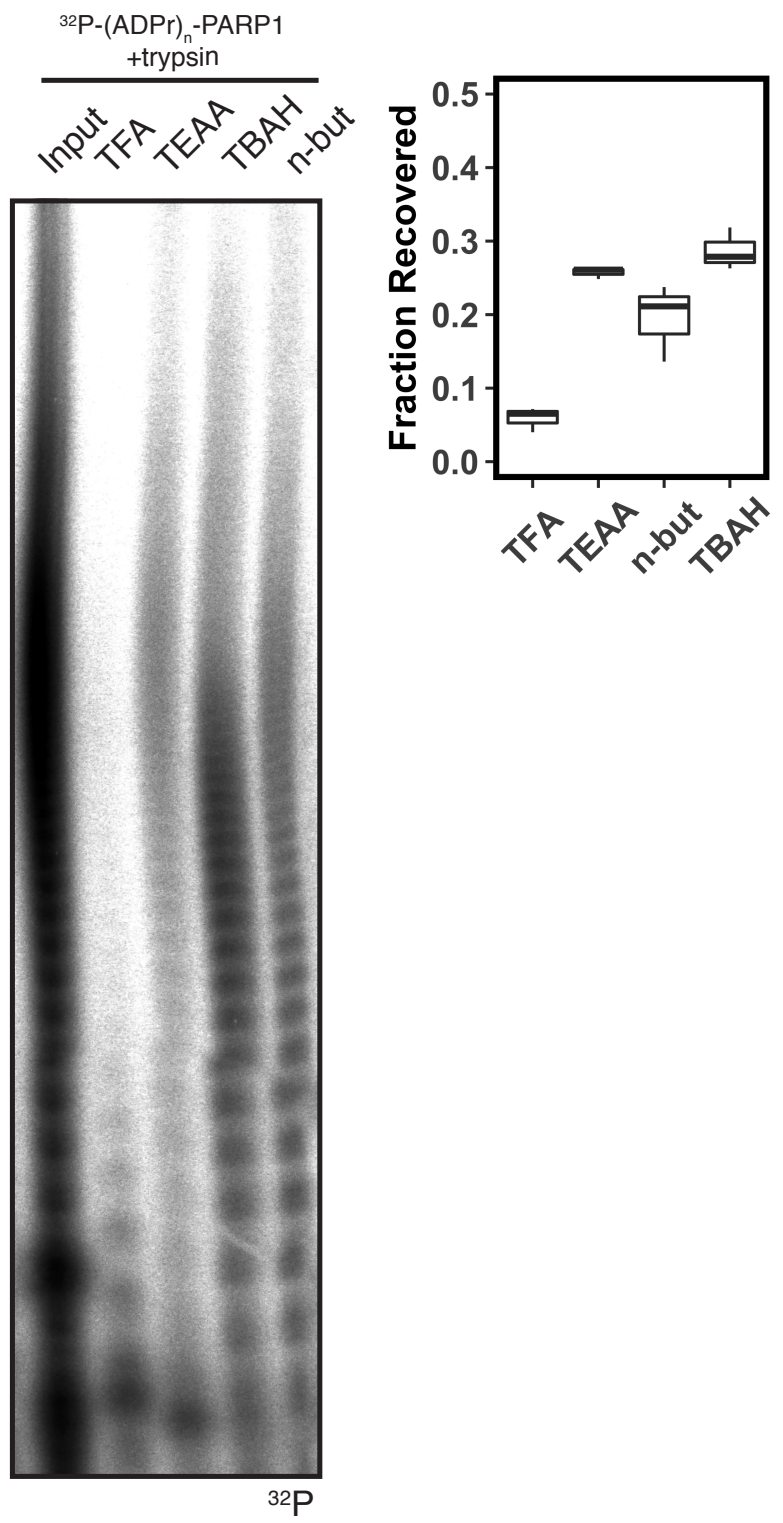

Supplementary Figure 1. Solid-phase extraction with cationic ion-pairing reagents improves recovery of ADP-ribosylated peptides. (A) Urea-PAGE analyses of ${ }^{32} \mathrm{P}-$ $(\text { ADPr })_{n}$-PARP1 digested with trypsin before (Input), and after solid-phase extraction on a $\mathrm{C}_{18}$ cartridge using trifluoroacetic acid (TFA), triethylammonium acetate (TEAA), n-butylamine (n-but), tetrabutylammonium hydroxide (TBAH) as an ion-pairing reagent in the mobile phase. Gel was analyzed by autoradiography. (B) Relative recovery of radioactive species in (A) after solid-phase extraction. Radioactive counts were acquired by scintillation counting, and the relative recovery was calculated as a fraction of the input $(n=3)$. 